International Journal of Pure and Applied Mathematics

Volume $91 \quad$ No. 3 2014, 381-387

ISSN: $1311-8080$ (printed version); ISSN: 1314-3395 (on-line version)

url: http://www.ijpam.eu

doi: http://dx.doi.org/10.12732/ijpam.v91i3.11

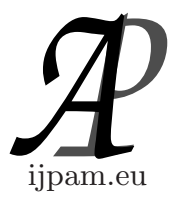

\title{
ON THE RANKS OF POINTS OF TANGENT DEVELOPABLES (SCROLLS AND SEGRE-VERONESE VARIETIES)
}

\author{
E. Ballico \\ Department of Mathematics \\ University of Trento \\ 38123 Povo (Trento) - Via Sommarive, 14, ITALY
}

\begin{abstract}
We compute the X-rank of points of the tangent developable of embeddings of the Hirzebruch surfaces.
\end{abstract}

AMS Subject Classification: 14N05, 14Q05, 15A69

Key Words: $X$-rank, tangent developable, generic tangent space

\section{Introduction}

For any integral variety $X \subset \mathbb{P}^{r}$ defined over an algebraically closed field $\mathbb{K}$ let $\tau(X) \subseteq \mathbb{P}^{r}$ denote the tangent developable of $X$, i.e. the closure in $\mathbb{P}^{r}$ of all Zariski tangent spaces $T_{P} X \subset \mathbb{P}^{r}, P \in X_{\text {reg. }}$. For each $P \in \mathbb{P}^{r}$ the $X$-rank $r_{X}(P)$ of $P$ is the minimal cardinality of a set $A \subset X$ such that $P \in\langle A\rangle$, where \langle\rangle denote the linear span $([7])$.

For all integers $a>0$ and $b>0$ let $\nu_{a, b}: \mathbb{P}^{1} \times \mathbb{P}^{1} \rightarrow \mathbb{P}^{N}, N=a b+a+b$, denote the Segre-Veronese embedding of $\mathbb{P}^{1} \times \mathbb{P}^{1}$, i.e. the embedding of $\mathbb{P}^{1} \times \mathbb{P}^{1}$ induced by the complete linear system $\left|\mathcal{O}_{\mathbb{P}^{1} \times \mathbb{P}^{1}}(a, b)\right|$.

Theorem 1. Fix positive integers $a, b$ and assume that either $\operatorname{char}(\mathbb{K})=$ 0 or $\operatorname{char}(\mathbb{K}) \neq 2$ or $\operatorname{char}(K)=2, a \neq 2, b \neq 2$ and $(a, b) \neq(1,1)$. Fix $P \in \tau\left(\nu_{a, b}\left(\mathbb{P}^{1} \times \mathbb{P}^{1}\right)\right) \backslash \nu_{a, b}\left(\mathbb{P}^{1} \times \mathbb{P}^{1}\right)$. Let $Z \subset \mathbb{P}^{1} \times \mathbb{P}^{1}$ be the degree two zero-dimensional scheme such that $P \in\left\langle\nu_{a, b}(Z)\right\rangle$. Set $\rho:=r_{\nu_{a, b}\left(\mathbb{P}^{1} \times \mathbb{P}^{1}\right)}(P)$.

(i) If $Z$ is contained in some $D \in\left|\mathcal{O}_{\mathbb{P}^{1} \times \mathbb{P}^{1}}(1,0)\right|$, then $\rho=b$.

Received: December 12, 2013

(C) 2014 Academic Publications, Ltd. url: www.acadpubl.eu 
(ii) If $Z$ is contained in some $E \in\left|\mathcal{O}_{\mathbb{P}^{1} \times \mathbb{P}^{1}}(0,1)\right|$, then $\rho=a$.

(iii) In all other cases we have $\rho=a+b$.

If $\operatorname{char}(\mathbb{K})=2$, then it is easy to check that in cases (i), (ii), (iii) the rank of $P$ is 3 in case (i) if $b=2$, in case (ii) if $a=2$ and in case (iii) if $(a, b)=(1,1)$.

Theorem 2. Fix integers $e>0, a>0$ and $b \geq a e$. Assume either $\operatorname{char}(\mathbb{K})=0$ or $\operatorname{char}(\mathbb{K})>b$. Let $X_{e, a, b} \subset \mathbb{P}^{r}$ be the image of $F_{e}$ by the morphism $\phi$ induced by the complete linear system $\left|\mathcal{O}_{F_{e}}(a h+b f)\right|$. Fix $P \in$ $\left.\tau\left(X_{e, a, b}\right) \backslash X_{e, a, b}\right)$; if $b=e a$ assume the existence of $v \subset F_{e} \backslash h$ such that $\operatorname{deg}(v)=2$ and $P \in\langle\phi(v)\rangle$. There is a degree 2 connected scheme $v \subset F_{e}$ such that $P \in\langle\phi(v)\rangle$. Set $O:=v_{\text {red }}$. Let $F$ be the fiber of the ruling of $F_{e}$ containing $O$. Set $\rho:=r_{X_{e, a, b}}(P)$.

(i) If $v \subset F$, then $\rho=a$,.

(ii) If $v \nsubseteq F$ and $O \notin h$, then $\rho=b$.

(iii) If $v \nsubseteq F, O \in h$ and $v \nsubseteq h$, then $\rho=b-e a+a$.

(iv) Assume $v \subset h$. Then $\rho=b-e a$.

Is concision true for Segre-Veronese embeddings? More precisely we may ask it in a weak form (part (a) of Question 1) and in a strong form (part (b) of Question 1).

Question 1. Fix integers $n \geq 2$ and $d_{i} \geq 1, r_{i} \geq m_{i} \geq 0,1 \leq i \leq$ $n$. Fix linear subspaces $M_{i} \subseteq \mathbb{P}^{r_{i}}$. Let $u: \mathbb{P}^{m_{1}} \times \cdots \times \mathbb{P}^{m_{n}} \rightarrow \mathbb{P}^{N}, N:=$ $-1+\prod_{i=1}^{n}\left(\begin{array}{c}m_{i}+d_{i} \\ m_{i}\end{array}\right)$, be the Segre-Veronese embedding of $\mathbb{P}^{m_{1}} \times \cdots \times \mathbb{P}^{m_{n}} \mathbb{P}^{r_{n}}$ of multidegree $\left(d_{1}, \ldots, d_{n}\right)$. Set $X:=u\left(\mathbb{P}^{m_{1}} \times \cdots \times \mathbb{P}^{m_{n}} \mathbb{P}^{r_{n}}\right)$ and $Y:=u\left(M_{1} \times\right.$ $\left.\cdots \times M_{n}\right)$. Fix $P \in\langle Y\rangle$ ?

(a) Is $r_{X}(P)=r_{Y}(P)$ ?

(b) If (a) is true for $P$ is every set $S \subset X$ evincing the $X$-rank of $P$ contained in $Y$ ?

If part (a) of Question 1 is true, then we may use Theorem 1 for SegreVeronese embeddings.

\section{The Proofs}

For any smooth surface $X$, every effective divisor $D \subset X$ and every zerodimensional scheme $Z \subset X$ let $\operatorname{Res}_{D}(Z)$ denote the residual scheme of $Z$ with respect to $D$, i.e. the closed subscheme of $X$ with $\mathcal{I}_{X}: \mathcal{I}_{D}$ as its ideal sheaf. 
Lemma 1. Fix integers $a \geq 0$ and $b \geq 0$. Let $Z \subset \mathbb{P}^{1} \times \mathbb{P}^{1}$ be a zerodimensional scheme such that $\operatorname{deg}(Z) \leq a+b+1$. We have $h^{1}\left(\mathcal{I}_{Z}(a, b)\right)>0$ if and only if either there is $D \in\left|\mathcal{O}_{\mathbb{P}^{1} \times \mathbb{P}^{1}}(1,0)\right| \operatorname{such}$ that $\operatorname{deg}(Z \cap D) \geq b+2$ or there is $E \in\left|\mathcal{O}_{\mathbb{P}^{1} \times \mathbb{P}^{1}}(0,1)\right|$ such that $\operatorname{deg}(E \cap Z) \geq a+2$.

Proof. Since the "if " part is obvious by the cohomology of line bundles on $D$ and $E$, it is sufficient to prove the "only if" part. We use induction on the integer $a+b$, the case $(a, b)=(0,0)$ being obvious, since $h^{1}\left(\mathcal{I}_{P}\right)=0$ for all $P \in \mathbb{P}^{1} \times \mathbb{P}^{1}$. Similarly, we conclude if either $a=0$ or $b=0$. Hence we may assume $a>0$ and $b>0$. Let $\alpha$ be the maximum of all integers $\operatorname{deg}(Z \cap D)$ for all $D \in\left|\mathcal{O}_{\mathbb{P}^{1} \times \mathbb{P}^{1}}(1,0)\right|$ and $\beta$ the maximum of all integers $\operatorname{deg}(E \cap Z)$ for all $E \in$ $\left|\mathcal{O}_{\mathbb{P}^{1} \times \mathbb{P}^{1}}(0,1)\right|$. Assume $\alpha \leq b+1$ and $\beta \leq a+1$. Take $D \in\left|\mathcal{O}_{\mathbb{P}^{1} \times \mathbb{P}^{1}}(1,0)\right|$ such that $\operatorname{deg}(Z \cap D)=\alpha$. We have $\operatorname{deg}\left(\operatorname{Res}_{D}(Z)\right)=\operatorname{deg}(Z \cap D) \leq a+b-1$. Since $\operatorname{deg}(Z \cap D) \leq b+1$, we have $h^{1}\left(D, \mathcal{I}_{Z \cap D}(a, b)\right)=0$. Hence the Castelnuovo's sequence gives $h^{1}\left(\mathcal{I}_{\operatorname{Res}_{D}(Z)}(a-1, b)\right)>0$. By the inductive assumption either there is $T \in\left|\mathcal{O}_{\mathbb{P}^{1} \times \mathbb{P}^{1}}(1,0)\right|$ such that $\operatorname{deg}\left(\operatorname{Res}_{D}(Z) \cap T\right) \geq b+2$ or there is $E \in\left|\mathcal{O}_{\mathbb{P}^{1} \times \mathbb{P}^{1}}(0,1)\right|$ such that $\operatorname{deg}\left(E \cap \operatorname{Res}_{D}(Z)\right) \geq a+1$. The first case is impossible, because $Z \supseteq \operatorname{Res}_{D}(Z)$ and so $\operatorname{deg}\left(\operatorname{Res}_{D}(Z) \cap T\right) \leq \operatorname{deg}(Z \cap T) \leq \alpha$. Therefore the latter case occurs, i.e. $\beta=a+1$ and $D \cap Z \cap E=\emptyset$. Exchanging the role of the rulings we conclude by induction on $a+b$, unless $\alpha=b+1$. The scheme $Z \cap(D \cup E)$ has degree $a+b+2$ and hence $\operatorname{deg}(Z) \geq a+b+2$, a contradiction.

Lemma 2. Fix a rational normal curve $C \subset \mathbb{P}^{r}$ and $P \in \tau(C) \backslash C$. If $r=2$ and $\operatorname{char}(\mathbb{K})=2$, then either $r_{C}(P)=3$ or $r_{C}(P)=3$ and the latter occurs if and only if $P$ is the strange point of $C$. In all other cases we have $r_{C}(P)=r$.

Proof. Since $C$ is a smooth variety and $P \in \tau(C) \backslash C$, there is a degree two zero-dimensional connected scheme $Z \subset C$ such that $P \in\langle Z\rangle$. Since $P \notin C$, we have $P \notin\left\langle Z^{\prime}\right\rangle$ for any $Z^{\prime} \subsetneq Z$. Take $A \subset C$ evincing the $C$-rank of $P$. Since $Z$ is not reduced, we have $Z \neq A$. Hence $h^{1}\left(\mathcal{I}_{A \cup Z}(1)\right)>0([5]$, Lemma 1$)$. Since $C \cong \mathbb{P}^{1}$, the cohomology of line bundles on $\mathbb{P}^{1}$ gives $\operatorname{deg}(Z \cup A) \geq r+2$. Therefore $r_{C}(P)=\sharp(A) \geq r$. For degree reasons $P$ may be a strange point of $C$ only if $\operatorname{char}(K)=2=r$. If $P$ is not a strange point of $C$, then $r_{C}(P) \leq r([1])$. If $\operatorname{char}(\mathbb{K})=2=r$ and $P$ is the strange point of $C$, then $r_{C}(P)=3([1])$

Proof of Theorem 1. The scheme $Z$ exists, because $\nu_{a, b}\left(\mathbb{P}^{1} \times \mathbb{P}^{1}\right)$ is a smooth variety. In all cases the proof below easily check the uniqueness of $Z$; anyway, the proof works for any $Z$ without using its uniqueness. Set $\{O\}:=Z_{\text {red }}$. In the set-up of part (i) $Z$ is contained in a the linear span of the degree $b$ 
rational normal curve $\nu_{a, b}(D)$. Since $\left.r_{\nu_{a, b}(D)}(P)\right) \leq b$ (Lemma 2) we get the inequality $\rho \leq b$ in the set-up of part (i). In the same way the curve $r_{a, b}(E)$ gives the inequality $\rho \leq a$ in the set-up of (ii). Take the set-up of (iii). Since $\operatorname{dim}\left(\left|\mathcal{O}_{\mathbb{P}^{1} \times \mathbb{P}^{1}}(1,1)\right|\right)=3 \geq 2=\operatorname{deg}(Z)$, there is $C \in\left|\mathcal{O}_{\mathbb{P}^{1} \times \mathbb{P}^{1}}(1,1)\right|$ containing $Z$. Since $Z$ is neither as in case (i) nor in case (ii), $C$ is irreducible and hence $P \in\left\langle\nu_{a, b}(C)\right\rangle$ with $\nu_{a, b}(C)$ rational normal curve of degree $a+b$. Hence $\rho \leq a+b$ by Lemma 2 .

Fix $A$ evincing $r_{X}(P)$ and assume $\sharp(A) \leq a+b-1$. We have $h^{1}\left(\mathcal{I}_{Z}(a, b)\right)>0$ $\left(\left[5\right.\right.$, Lemma 1]). Set $\{O\}:=Z_{\text {red }}$. Set $W:=Z \cup A$. We have $h^{1}\left(\mathcal{I}_{A}(a, b)\right)=$ 0 and $h^{1}\left(\mathcal{I}_{W}(a, b)\right)>0$. Since $\operatorname{deg}(W) \leq a+b+1$, either there is $D \in$ $\left|\mathcal{O}_{\mathbb{P}^{1} \times \mathbb{P}^{1}}(1,0)\right|$ such that $\operatorname{deg}(D \cap W) \geq b+2$ or there is some $E \in\left|\mathcal{O}_{\mathbb{P}^{1} \times \mathbb{P}^{1}}(0,1)\right|$ $\operatorname{such}$ that $\operatorname{deg}(E \cap W) \geq a+2$ (Lemma 1). Assume for instance the existence of $D$. We need to prove that we are in case (i). Assume that $Z \nsubseteq D$. Since $A$ evinces $\rho, \nu_{a, b}(A)$ is linearly independent. Hence $\sharp(A \cap D) \leq b+1$. Since $Z \nsubseteq D$, we get $O \in D, O \notin A$ and $\sharp(A \cap D)=d+1$. We have $\operatorname{deg}\left(\operatorname{Res}_{D}(W)\right)=\operatorname{deg}(W)-$ $b-2$. Assume for the moment $h^{1}\left(\mathcal{I}_{\operatorname{Res}_{D}(W)}(a-1, b)\right)=0$. By [6, Lemma 5.1] we get $\operatorname{Res}_{D}(Z)=A \backslash A \cap D$. Since $\operatorname{Res}_{D}(Z)=\{O\} \nsubseteq A$, we get a contradiction. Now assume $h^{1}\left(\mathcal{I}_{\operatorname{Res}_{D}(W)}(a-1, b)\right)>0$. Since $\operatorname{deg}(W) \leq(a-1)+b$, either there is $D^{\prime} \in\left|\mathcal{O}_{\mathbb{P}^{1} \times \mathbb{P}^{1}}(1,0)\right|$ such that $\operatorname{deg}\left(D^{\prime} \cap \operatorname{Res}_{D}(W)\right) \geq b+2$ or there is some $E^{\prime} \in\left|\mathcal{O}_{\mathbb{P}^{1} \times \mathbb{P}^{1}}(0,1)\right|$ such that $\operatorname{deg}\left(E^{\prime} \cap \operatorname{Res}_{D}(W)\right) \geq a+1$ (Lemma 1). First assume the existence of $D^{\prime}$. Since $\operatorname{Res}_{D}(W)=\{O\} \cup(A \backslash A \cap D)$, we have $D^{\prime} \neq D$. Hence $\sharp\left(A \cap D^{\prime}\right) \geq b+2$. Hence $\nu_{a, b}(A)$ is linearly dependent, a contradiction. Now assume the existence of $E^{\prime}$. Since $\operatorname{deg}\left(\{O\} \cap E^{\prime}\right) \leq 1$ we get $\sharp\left(A \cap E^{\prime}\right) \geq a$. Since $\operatorname{deg}\left(D \cap E^{\prime}\right)=1$, we get $\sharp(A) \geq b+2+a-1=a+b+1$, a contradiction.

Lemma 3. Fix integers $e>0, a>0$ and $b \geq a e$. Fix a degree 2 connected scheme $v \subset F_{e}$ and set $O:=v_{\text {red }}$. If $O \in h$, then assume $b>$ ae. Let $A \subset F_{e}$ be any finite set such that $h^{1}\left(\mathcal{I}_{v \cup A}(a h+b f)\right)>h^{1}\left(\mathcal{I}_{\{O\} \cup A}(a h+b f)\right)$ and with minimal cardinality, $\rho$, among all such finite sets. Let $F$ be the fiber of the ruling of $F_{e}$ containing $O$.

(i) If $v \subset F$, then $\rho=a, O \notin A$ and $A \subset F$.

(ii) If $v \nsubseteq F$ and $O \notin h$, then $\rho=b$.

(iii) If $v \nsubseteq F, O \in h$ and $v \nsubseteq h$, then $\rho=b-e a+a$.

(iv) If $v \subset h$, then $\rho=b-e a$.

Proof. It is easy to check that the minimality condition for the set $A$ implies $O \notin A, h^{1}\left(\mathcal{I}_{A \cup\{O\}}(a h+b f)\right)=0$, and $h^{1}\left(\mathcal{I}_{v \cup A}(a h+b f)\right)=1$. Let $\pi: F_{e} \rightarrow \mathbb{P}^{1}$ denote the ruling of $F_{e}$. 
(a) In this step we prove that in each case (i), (ii), (iii) and (iv) the integer $\rho$ is is at most the one claimed in the statement of the lemma. Take $v$ as in case (i). For each $E \subset F \backslash\{O\}$ with $\sharp(E)=a$ we have $h^{1}\left(F, \mathcal{I}_{E \cup v}(a h+b f)\right)=1$ and $h^{1}\left(F, \mathcal{I}_{\{O\} \cup E}(a h+b f)\right)=0$. Since $b \geq e a$, we have $h^{1}\left(\mathcal{O}_{F_{e}}(a h+(b-1) f)\right)=0$. Hence $h^{1}\left(\mathcal{I}_{E \cup v}(a h+b f)\right)=1$ and $h^{1}\left(\mathcal{I}_{\{O\} \cup E}(a h+b f)\right)=0$. Therefore $\rho \leq a$. Take $v$ as in case (ii). Since $O \notin h$, the linear system $\left|\mathcal{O}_{F_{e}}(h+e f)\right|$ induces an embedding at $O$ and it injective outside $h$. Take a general $C \in \mid \mathcal{I}_{v}(h+$ ef $) \mid$. Since $v \nsubseteq F$, a dimensional count gives the irreducibility of $C$. Hence $C \cong \mathbb{P}^{1}$. Since $\operatorname{deg}\left(\mathcal{O}_{C}(a h+b f)\right)=b$, we have $h^{1}\left(C, \mathcal{I}_{E \cup v}(a h+b f)\right)=1$ and $h^{1}\left(C, \mathcal{I}_{v \cup E}(a h+b f)\right)=0$ for all $E \subset C \backslash\{O\}$ with $\sharp(E)=b$. Since $h^{1}\left(\mathcal{O}_{F_{e}}((a-1) h+(b-e) f)\right)=0$, we get $\rho \leq b$. In case (iv) we use the curve $h \cong \mathbb{P}^{1}$. In case (iii) we use the curve $h \cup F$ with $E \subset h \cup F \backslash\{O\}, \sharp(E \cap h)=b-e a$ and $\sharp(E \cap F)=a$; indeed, we have $h^{0}\left(h \cup F, \mathcal{O}_{h \cup F}(a h+b f)\right)=b-e a+a$.

(b) Take the set-up of (ii) and take any $E \subset F_{e}$ with $\sharp(E) \leq b-1$ and $h^{1}\left(\mathcal{I}_{\{O\} \cup E}(a h+b f)\right)=0$. We need to prove that $h^{1}\left(\mathcal{I}_{v \cup E}(a h+b f)\right)=0$. Assume $h^{1}\left(\mathcal{I}_{v \cup E}(a h+b f)\right)>0$. Set $B_{0}:=\{O\} \cup E$. Take $D_{1} \in\left|\mathcal{O}_{F_{e}}(f)\right|$ such that $a_{1}:=\sharp\left(B_{0} \cap D_{1}\right)$ is maximal and set $B_{1}:=B_{0} \backslash D_{1}$. For all $i=2, \ldots, b$ define recursively $D_{i}, a_{i}$ and $B_{i}$ in the following way Take $D_{i} \in\left|\mathcal{O}_{F_{e}}(f)\right|$ such that $a_{i}:=\sharp\left(B_{i-1} \cap D_{i}\right)$ is maximal with the only restriction that $D_{i} \neq D_{j}$ for $j<i$ if $B_{i-1}=\emptyset$. Set $B_{i}:=B_{i-1} \backslash B_{i-1} \cap B_{i-1} \cap D_{i}$. The sequence $a_{1}, \ldots, a_{b}$ is non-increasing. Since $\sharp(B) \leq b$, we have $B_{b}=\emptyset$ and $a_{1}+\cdots+a_{b}=\sharp(B)$. Set $T:=a h+D_{1}+\cdots+D_{b}$. We have $B \subset T$, but $v \nsubseteq T$, because $F$ appears only once in the sequence $D_{1}, \ldots, D_{b}$ and $O \notin h$. Hence $\operatorname{Res}_{T}(A \cup v)=\{O\}$. Hence $h^{1}\left(\mathcal{I}_{\operatorname{Res}_{T}(v \cup A)}\right)=0$. Since $h^{1}\left(\mathcal{I}_{B}(a h+b f)\right)=0$, the Castelnuovo's sequence (e.g. [2], eq. (1)) gives a contradiction.

(c) Take the set-up of (iv). Hence $b>a e$. Take any $E \subset F_{e}$ such that $h^{1}\left(\mathcal{I}_{\{O\} \cup E}(a h+b f)\right)=0, \sharp(E) \leq b-e a-1, O \notin E, h^{1}\left(\mathcal{I}_{v \cup E}(a h+b f)\right)>$ 0 and $h^{1}\left(\mathcal{I}_{v \cup E^{\prime}}(a h+b f)\right)=0$ for all $E^{\prime} \subsetneq E$. Take $B_{0}:=\{O\} \cup E$ and make the construction $a_{i}, D_{i}, B_{i}$ as in step (b). Let $x$ be the minimal integer such that $a_{x+1}=0$. In the set-up of (iv) we have $x \leq b-e b$. Set $T^{\prime}:=$ $h \cup D_{1} \cup \cdots \cup D_{x-1}$. Since $v \cup E \subset T^{\prime} \cup D_{x}$, we have $\operatorname{Res}_{T^{\prime}}(v \cup E) \subset D_{x}$. Since $v \subset h \subset T^{\prime}$, we have $\operatorname{Res}_{T^{\prime}}(v \cup E)=D_{x} \cap\left(E \backslash E \cap D_{x} \cap h\right)$. First assume $x \geq 2$ and $1 \leq \sharp\left(E \backslash E \cap D_{x} \cap h\right) \leq a$. Since $\operatorname{Res}_{T^{\prime}}(v \cup E) \neq \emptyset$, minimality of $A$ gives $h^{1}\left(\mathcal{I}_{(v \cup E) \cap T^{\prime}}(a h+b f)\right)=0$. The Castelnuovo' sequence gives $h^{1}\left(\mathcal{I}_{\operatorname{Res}_{T^{\prime}}(v \cup E)}((a-1) h+(b-x) f)\right)>0$. Since $\sharp\left(\operatorname{Res}_{T^{\prime}}(v \cup E)\right) \leq a$ and $\left.\operatorname{Res}_{T^{\prime}}(v \cup E) \cap h\right)=\emptyset$, we get a contradiction. Now assume $x \geq 2$ and $\sharp\left(E \backslash E \cap D_{x} \cap h\right) \geq a+1$. Since $h^{1}\left(\mathcal{I}_{E}(a h+b f)\right)=0$, we get $a_{x}=a+1$ and that $D_{x} \cap E \cap h=\emptyset$. Since the sequence $a_{i}$ is non-decreasing, we get 
$a_{i}=a+1$ for all $i \leq x$ and hence $\sharp(B)=x(a+1)$. In this case we may exchange the role of the curves $D_{i}, 1 \leq i \leq x$, and win if there is at least one $i \in\{1, \ldots, x\}$ such that $D_{i} \cap A \cap h \neq \emptyset$. Since $O \notin E$, the curve $D_{i}$ containing $O$ has this property. Now assume $x=1$. Since $O \in B$, we get $D_{1}=F$. Since $h^{1}\left(\mathcal{I}_{\{O\} \cup E}(a h+b f)\right)=0$, we have $\sharp(A) \leq a$. Since $b>a e$ and $h^{1}\left(\mathcal{O}_{F_{e}}((a-1) h+(b-1) f)\right)=0$, the linear system $\left|\mathcal{O}_{F_{e}}(a h+b f)\right|$ gives an embedding $\phi$ of the curve $h \cup F$ with $\langle\phi(h+F)\rangle \cong \mathbb{P}^{a+b-e a},\langle\phi(h)\rangle=\mathbb{P}^{a}$, $\langle\phi(h)\rangle=\mathbb{P}^{b-e a}$ and $\phi(h), \phi(F)$ rational normal curves in their linear span. Since $p_{a}(h+F)=0$, we get $h^{1}\left(\mathcal{I}_{v \cup F}(a h+b f)\right)=0$, a contradiction.

(d) Take the set-up of (iii). Hence $b>a e$. Take any $E \subset F_{e}$ such that $h^{1}\left(\mathcal{I}_{\{O\} \cup E}(a h+b f)\right)=0, \sharp(E) \leq b-e a-1, O \notin E, h^{1}\left(\mathcal{I}_{v \cup E}(a h+b f)\right)>0$ and $h^{1}\left(\mathcal{I}_{v \cup E^{\prime}}(a h+b f)\right)=0$ for all $E^{\prime} \subsetneq E$. Take $B_{0}:=\{O\} \cup E$ and make the construction $a_{i}, D_{i}, B_{i}$ as in steps (b) and (c). Let $x$ be the minimal integer such that $a_{x+1}=0$. In the set-up of (iii) we have $x \leq b-e b-a$. Let $c \in\{1, \ldots, x\}$ be the integer such that $D_{c}=F$. First assume $a_{c} \leq a$. Take $T:=h \cup\left(\bigcup_{i \neq c} D_{i}\right)$. We have $\operatorname{Res}_{T}(v \cup E)=D_{c} \cap B$, because $\left.\operatorname{Res}_{T}(v)\right)=\{O\}$. We conclude as in step (c). Now assume $a_{c}=a+1$. If $c<x$ and $a_{x} \leq a$, then we conclude as in step (c) using $T^{\prime}$ instead of $T$, because $v \subset F \cup h \subset T^{\prime}$. Hence we may assume $a_{i}=a+1$ for all $i$. Let $J:=D_{1} \cup \cdots \cup D_{x}$. Since $F \subseteq J$ and $v \nsubseteq F$, we have $\left.\operatorname{Res}_{v \cup J}(v \cup E)\right)=\{O\}$. Hence $(v \cup E) \cap J \neq v \cup A$. Hence $h^{1}\left(\mathcal{I}_{(v \cup E) \cap J}(a h+b f)\right)=0$. Since $(a+1) x \leq b-e a+a$, we have $b-x \geq e a$. Hence $\mathcal{O}_{F_{e}}(a h+(b-x) f)$ is spanned. Therefore $h^{1}\left(\mathcal{I}_{\{O\}}(a h+(b-x) f)\right)=0$. The Castelnuovo's sequence gives $h^{1}\left(\mathcal{I}_{v \cup E}(a h+b f)\right)=0$, a contradiction.

Proof of Theorem 2. In the case $b=a e$ we may assume $b>e a$. Use Lemma 3 to get that $\rho$ is at least the value claimed in the statement of Theorem 2. In all cases we get the opposite inequality is true using [1] and the curves introduced in step (a) of the proof of Lemma 3.

\section{Acknowledgements}

The author was partially supported by MIUR and GNSAGA of INdAM (Italy).

\section{References}

[1] E. Ballico, An upper bound for the X-ranks of points of $\mathbb{P}^{n}$ in positive characteristic. Albanian J. Math. 5 (2011), no. 1, 3-10. 
[2] , E. Ballico, On the generic rank of linear spans of tangent vectors, preprint.

[3] E. Ballico, An Upper Bound for the Symmetric Tensor Rank of a Low Degree Polynomial in a Large Number of Variables, Hindawi Publishing Corporation Geometry Volume 2013, Article ID 715907, 2 pages http://dx.doi.org/10.1155/2013/715907

[4] E. Ballico, Symmetric Tensor Rank and Scheme Rank: An Upper Bound in terms of Secant Varieties, Geometry Volume 2013, Article ID 614195, 3 pages http://dx.doi.org/10.1155/2013/614195

[5] E. Ballico and A. Bernardi, Decomposition of homogeneous polynomials with low rank, Math. Z. 271 (2012) 1141-1149.

[6] E. Ballico and A. Bernardi, Stratification of the fourth secant variety of Veronese variety via the symmetric rank, Adv. Pure Appl. Math. 4 (2013), no. 2, 215-250; DOI: 10.1515/apam-2013-0015

[7] J. M. Landsberg, Tensors: Geometry and Applications. Graduate Studies in Mathematics, Vol. 128, Amer. Math. Soc. Providence, 2012. 
